\title{
A prime/boost vaccine platform efficiently identifies CD27 agonism and depletion of myeloid-derived suppressor cells as therapies that rationally combine with checkpoint blockade in ovarian cancer
}

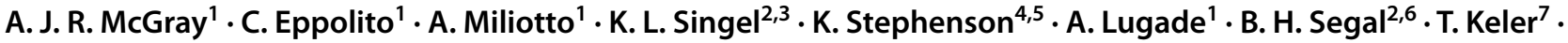 \\ G. Webster ${ }^{8} \cdot$ B. Lichty ${ }^{4} \cdot$ D. Kozbor ${ }^{2} \cdot$ K. Odunsi ${ }^{1,9}$
}

Received: 24 November 2020 / Accepted: 31 March 2021 / Published online: 20 April 2021

(C) The Author(s), under exclusive licence to Springer-Verlag GmbH Germany, part of Springer Nature 2021

\begin{abstract}
Cancer immunotherapies have generated remarkable clinical responses for some patients with advanced/metastatic disease, prompting exploration of rational combination therapies to bolster anti-tumor immunity in patients with limited response or those who experience tumor progression following an initial response to immunotherapy. In contrast to other tumor indications, objective response rates to single-agent PD-1/PD-L1 blockade in ovarian cancer are limited, suggesting a need to identify combinatorial approaches that lead to tumor regression in a setting where checkpoint blockade alone is ineffective. Using a pre-clinical model of aggressive intraperitoneal ovarian cancer, we have previously reported on a heterologous prime/boost cancer vaccine that elicits robust anti-tumor immunity, prolongs survival of tumor-bearing mice, and which is further improved when combined with checkpoint blockade. As tumor control in this model is CD8 + T cell dependent, we reasoned that the prime/boost vaccine platform could be used to explore additional treatment combinations intended to bolster the effects of CD8 $+\mathrm{T}$ cells. Using whole tumor transcriptomic data, we identified candidate therapeutic targets anticipated to rationally combine with prime/boost vaccination. In the context of a highly effective cancer vaccine, CD27 agonism or antibody-mediated depletion of granulocytic cells each modestly increased tumor control following vaccination, with anti-PD-1 therapy further improving treatment efficacy. These findings support the use of immunotherapies with well-defined mechanisms(s) of action as a valuable platform for identifying candidate combination approaches for further therapeutic testing in ovarian cancer.
\end{abstract}

Keywords Ovarian cancer $\cdot$ Cancer vaccine $\cdot$ Cancer immunotherapy $\cdot$ CD27 $\cdot$ Suppressive myeloid cells $\cdot$ MDSC

This article was prepared, while Kelly L Singel was employed at Roswell Park Comprehensive Cancer Center. The opinions expressed in this article are the author's own and do not reflect the view of the National Institutes of Health, the Department of Health and Human Services, or the United States Government.

A. J. R. McGray

ajrobert.mcgray@ roswellpark.org

$\triangle$ K. Odunsi

odunsia@bsd.uchicago.edu

1 Center for Immunotherapy, Roswell Park Comprehensive Cancer Center, Roswell Park Cancer Institute, Elm and Carlton Sts, Buffalo, NY 14263, USA

2 Department of Immunology, Roswell Park Comprehensive Cancer Center, Buffalo, NY, USA

3 Office of Evaluation, Performance, and Reporting, National Institutes of Health, Bethesda, MD, USA

4 McMaster Immunology Research Centre, McMaster University, Hamilton, ON, Canada
5 Turnstone Biologics, Ottawa, ON, Canada

6 Department of Internal Medicine, Roswell Park Comprehensive Cancer Center and Jacobs School of Medicine and Biomedical Sciences, University At Buffalo, Buffalo, NY, USA

7 Celldex Therapeutics, Hampton, NJ, USA

8 Innate Immunotherapeutics, Auckland, New Zealand

9 Present Address: University of Chicago Comprehensive Cancer Center, 5841 South Maryland Avenue, MC1140, Chicago, IL 60637, USA 


\section{Introduction}

Cancer immunotherapies have demonstrated impressive clinical activity in some patients with advanced and metastatic cancer. As such, identifying immunological features of tumors that inform rational combination therapies, improve clinical response rates, and produce complete and durable tumor regression is actively being pursued. Tumors are equipped, either intrinsically or via adaptive resistance mechanisms [1, 2], to evade immune attack and limit immunotherapy effectiveness. Moreover, diverse mechanisms of therapeutic failure following immune checkpoint blockade (ICB) have emerged, including loss of immunogenic antigens, impaired antigen processing/presentation, tumor infiltration by suppressive cells (eg. MDSC or Tregs), upregulation of additional checkpoint receptors by $\mathrm{T}$ cells, and mutations in JAK/STAT signaling [3]. Therefore, identifying treatment modalities that synergize with ICB to enhance therapeutic response when ICB alone is ineffective, or which restore anti-tumor immunity following ICB failure are needed. This is particularly important in cancers with limited clinical response to ICB, including ovarian cancer, where objective responses to single-agent PD-1/PD-L1 blockade were only $10-15 \%$ among ovarian cancer patients in recent clinical trials [4].

Our group has focused extensively on therapeutic cancer vaccines to treat ovarian cancer patients [5-7]. In a recent report, we demonstrated using a pre-clinical intraperitoneal ovarian cancer model that a potent heterologous adjuvantbased prime/oncolytic virus boost vaccine significantly delayed tumor progression [8]. Therapeutic efficacy was further enhanced when vaccination was combined with an anti-PD-1 antibody, where anti-PD-1 reversed tumor-specific CD8 + T cell dysfunction in the tumor microenvironment (TME). As tumor control following vaccination in this model is CD8 + T cell dependent, we reasoned the prime/ boost vaccine could be used to identify and test additional combination therapies aimed at augmenting tumor-specific $\mathrm{CD} 8+\mathrm{T}$ cell activity. To this end, candidate therapeutic targets to test in combination with prime/boost vaccination were identified from tumor transcriptomic data [8] and combined with prime/boost vaccination alone or in combination with checkpoint blockade. A CD27 agonist antibody or antibody-mediated depletion of myeloid cells each modestly improved the already effective vaccine \pm anti-PD- 1 therapy, suggesting these pathways have relevance in enhancing the activity of tumor-specific CD $8+\mathrm{T}$ cells in ovarian cancer and warrant further exploration. These studies provide novel insights into strategies intended to enhance vaccine-induced CD8 + T cells, as well as the significance of the PD-1 pathway in regulating anti-tumor immunity.

\section{Results}

\section{CD27 agonism improves the impact of a potent prime/boost cancer vaccine}

We previously tested therapeutic prime/boost vaccination in the intraperitoneal murine IE9-mp1 ovarian cancer model, which expresses ovalbumin (OVA) as a model tumor antigen [8]. Combining the adjuvant-based MIS416 + OVA vaccine (MIS) with an oncolytic Maraba virus engineered to express OVA (MRB-OVA) as a vaccine booster (referred to as MIS/MRB) delayed tumor progression and was accompanied by upregulation of an intratumoral $\mathrm{T}$ cell signature that included the $\mathrm{T}$ cellassociated co-stimulatory receptor CD27 [8]. Further analysis of whole tumor transcriptomic data revealed that expression of CD70, the cognate CD27 ligand [9, 10], was generally low and did not increase following MIS alone or MIS/MRB (Fig. 1a), raising the possibility that insufficient CD27 co-stimulation impeded CD8 + T cell function. As OVA-specific CD8 + T cells expressed CD27 (albeit at reduced levels) following MIS/MRB (Fig. 1b), we reasoned that $\mathrm{CD} 27$ agonism might improve vaccine response by augmenting tumor-specific $\mathrm{CD} 8+\mathrm{T}$ cell expansion, persistence, and/or function. Agonist antiCD27 delivery (Fig. 1c) to coincide with CD8 $+\mathrm{T}$ cell expansion and peak response following MIS/MRB [8] delayed tumor progression (Fig. 1d) and improved survival (Fig. 1e).

\section{CD27 co-stimulation following vaccination does not overcome CD8 + T cell dysfunction in the TME and addition of PD-1 blockade further improves tumor control}

Increased therapeutic efficacy following MIS/MRB + antiCD27 correlated with a transient increase in circulating OVA-specific CD8 + T cells (Fig. 2a). However, cellular analysis 15 days following MRB-boosting revealed that while high frequencies of $\mathrm{T}$ cells were present within the TME, no change in the accumulation of intraperitoneal OVA-specific CD8 + tumor-associated lymphocytes (TALs), total CD3 + TILs (Fig. 2b and c), or clear functional enhancement of OVA-specific CD8 $+\mathrm{T}$ cells in either the periphery or TME (Fig. 2d) occurred as a result of $\mathrm{CD} 27$ co-stimulation. These findings suggested that CD27 agonism had only a transient impact and was insufficient to durably increase $\mathrm{T}$ cell accumulation or reverse $\mathrm{CD} 8+\mathrm{T}$ cell dysfunction in the ovarian TME. The elevated PD-1 expression on OVA-specific CD8 + TALs [8] was not impacted by CD27 agonism (Fig. 2e), and 
A

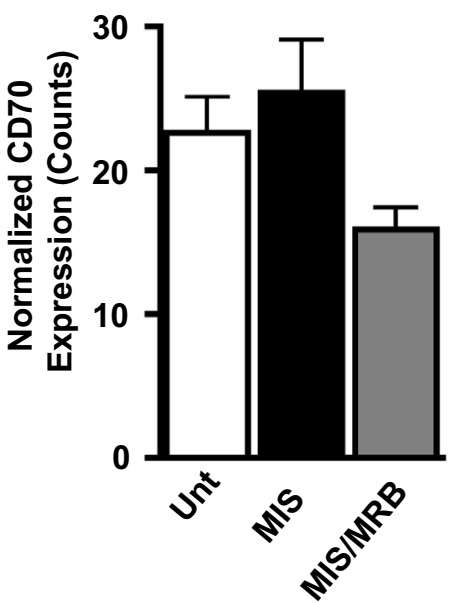

B

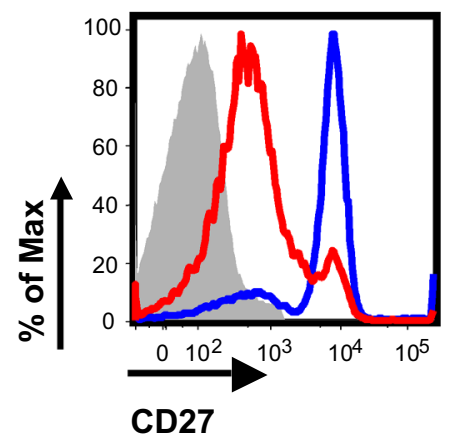

Gated on CD8+ T cells

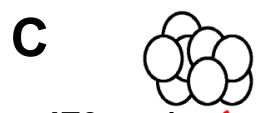

IE9-mp1
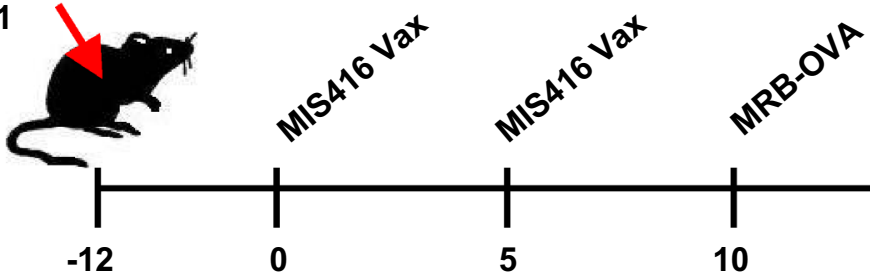

aCD27 or IgG Cont
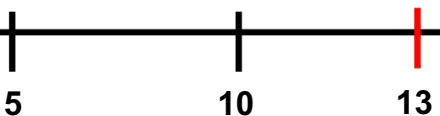

Days Post Vaccination

D

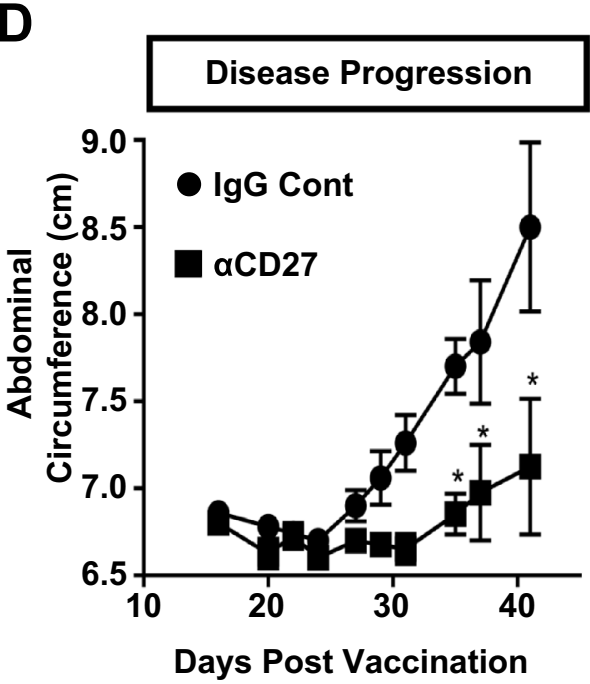

Fig. 1 CD27 co-stimulation improves therapeutic prime/boost vaccination. a Intratumoral expression of CD70 assessed by Nanostring following treatment $(n=8)$ b Representative FACs plot demonstrating relative CD27 expression on OVA-specific and non-specific CD8 + T cells following MIS/MRB Vaccination. c Schematic representation of experimental design and treatment schedule for MIS/MRB + anti-
$\mathbf{E}$

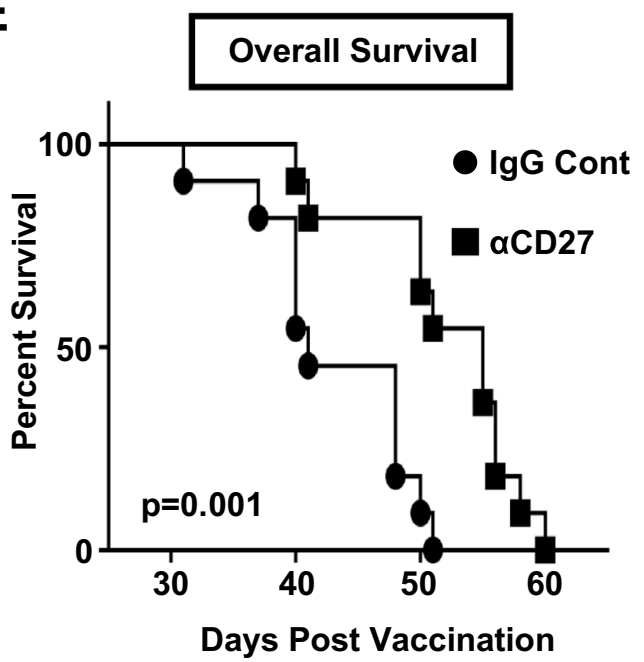

CD27/IgG Control. d IE9-mp1 tumor progression in mice following MIS/MRB combined with IgG Control (C) or anti-CD27 (a) $(n=4-5)$. e Compiled survival data for tumor-bearing mice as described in $\mathbf{d}(n=11)$. Data presented as mean \pm SEM. Data in $(\mathbf{d})$ are from one representative experiment 
A

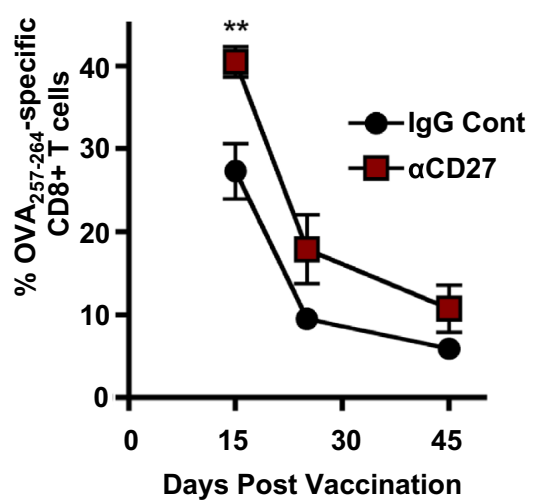

B

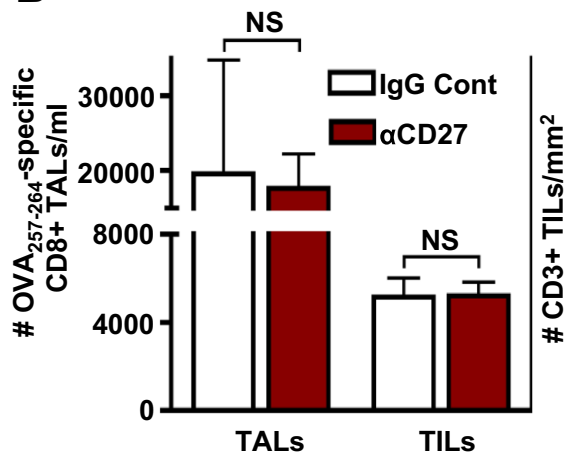

C
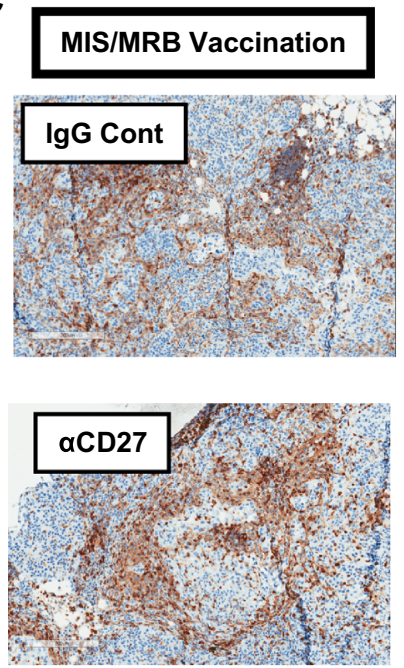

$\mathbf{E}$

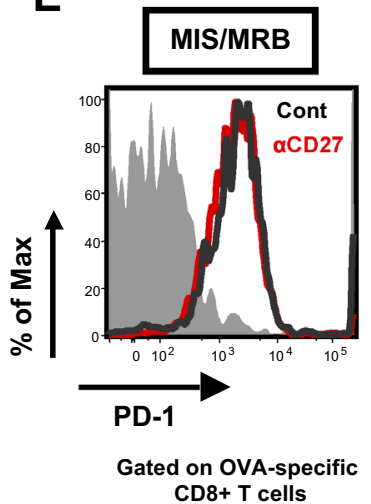

D
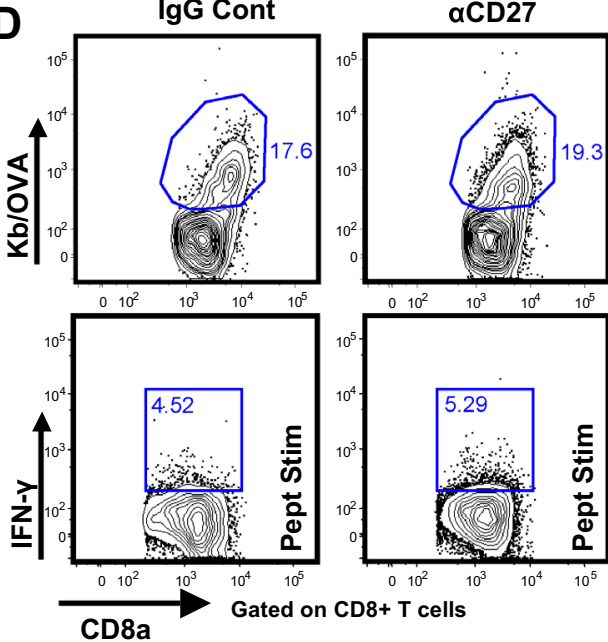

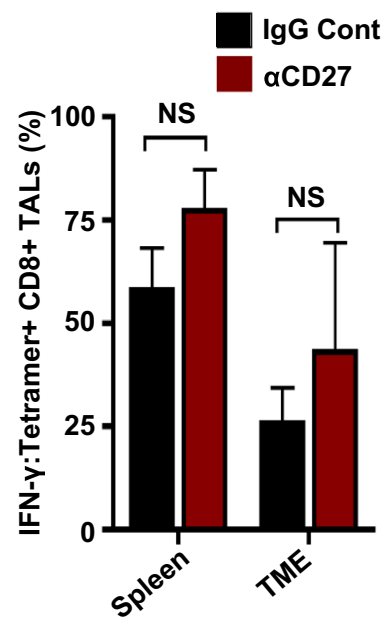

$\mathbf{F}$

MIS/MRB Vaccination

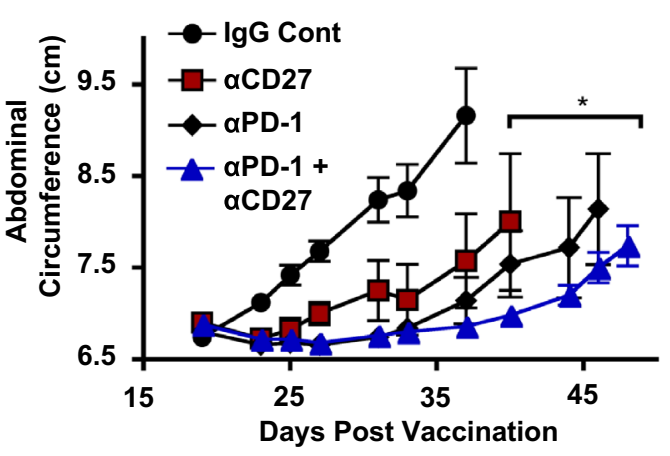

Fig. 2 CD27 co-stimulation following prime/boost vaccination is further enhanced by PD-1 blockade. a \% OVA-specific CD8+T cells were assessed in the blood at indicated time points post-vaccination with MIS/MRB combined with IgG (O) or anti-CD27 ( $(n=3-9 /$ time point). b OVA-specific CD8 + TALs were assessed in the peritoneal TME and CD3 + TILs in solid tumor lesions following MIS/ $\mathrm{MRB}+\mathrm{IgG}$ or anti-CD27 $(n=3)$. $\mathbf{c}$ Representative CD3 staining of tumors isolated from mice following treatment with MIS/MRB + IgG or anti-CD27 (Scale bar $=200 \mu \mathrm{m})$. d Left Panel: Representative FACs plots showing matched $\mathrm{OVA}_{257-264}$ tetramer staining and

ex vivo function of CD8 + TALs following $\mathrm{OVA}_{257-264}$ peptide stimulation. Right Panel: OVA-specific CD8 $+\mathrm{T}$ cell function assessed as a ratio of $\% \mathrm{IFN}-\gamma$ producing to tetramer $+\mathrm{CD} 8+\mathrm{T}$ cells following treatment in the spleen and TME $(n=3)$. Mice were treated with MIS/MRB + IgG or anti-CD27 as indicated. e Representative FACs plot comparing PD-1 expression on OVA-specific CD8 + TALs in the TME following MIS/MRB + IgG or anti-CD27. f IE9-mp1 tumor progression in mice following MIS/MRB combined with $\operatorname{IgG}(\mathbf{O})$, anti-

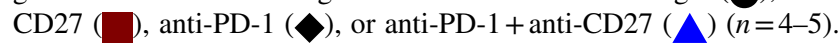
Data presented as mean \pm SEM. Data in (f) are from one experiment 
tumor control resulting from MIS/MRB + anti-CD27 was improved when anti-PD-1 therapy was added to the treatment regimen (Fig. 2f), although this combination was not significantly better than MIS/MRB + anti-PD-1 alone.

\section{Granulocytic cell accumulation negatively correlates with CD3 + TIL in human ovarian tumors}

In addition to targeting $\mathrm{T}$ cell co-stimulatory/co-inhibitory pathways, limiting intratumoral infiltration or function of suppressive immune cells can improve intratumoral $\mathrm{T}$ cell function. Tumor immune profiling of IE9-mp1 ovarian tumors suggested infiltration by diverse immune cell populations (including multiple $\mathrm{T}$ cell and myeloid cell subsets), which were further increased following prime/boost vaccination [8]. In line with this pre-clinical data, analysis of infiltrating immune cells from human ovarian cancer surgical specimens demonstrated abundant accumulation of $\mathrm{CD} 3+\mathrm{T}$ cells, as well as CD15 + granulocytic and CD14 + monocytic myeloid cells (Fig. 3a). Of note, CD3 + TIL frequency positively correlated with infiltration of CD14+monocytic cells, but negatively correlated with CD15 + granulocytic

A

\section{Human Ovarian Cancer - CD45+ Immune Infiltrate}

Sample ID:
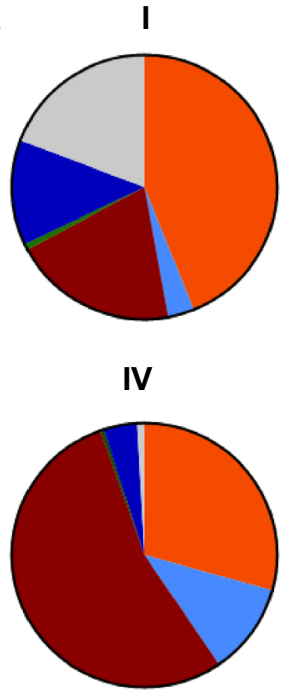
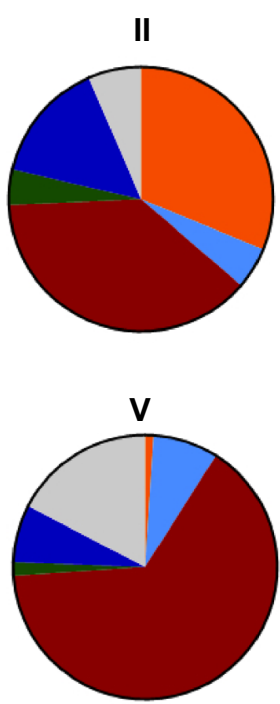

III
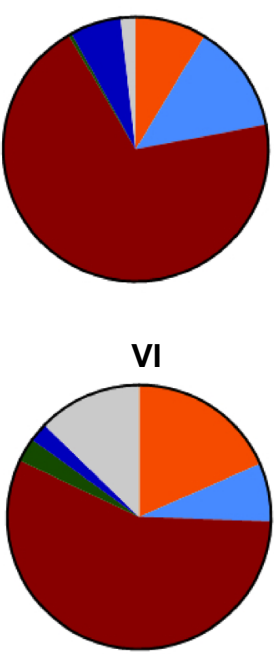

CD15+ Granulocytes

CD14+ Monocytes

CD3+ T cells

CD19+ B cells

CD56+CD3- NK Cells

Other CD45+ Cells

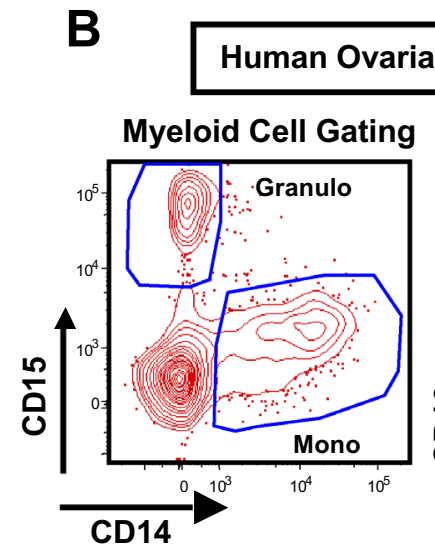

Gated on CD45+ Cells

Fig. 3 Analysis of immune infiltration in human ovarian cancer surgical specimens a Frequencies of indicated immune cell subsets (CD45 + Cells) were assessed in human ovarian cancer patient tumors $(n=6)$. b Representative gating of CD15 + granulocytic cells,
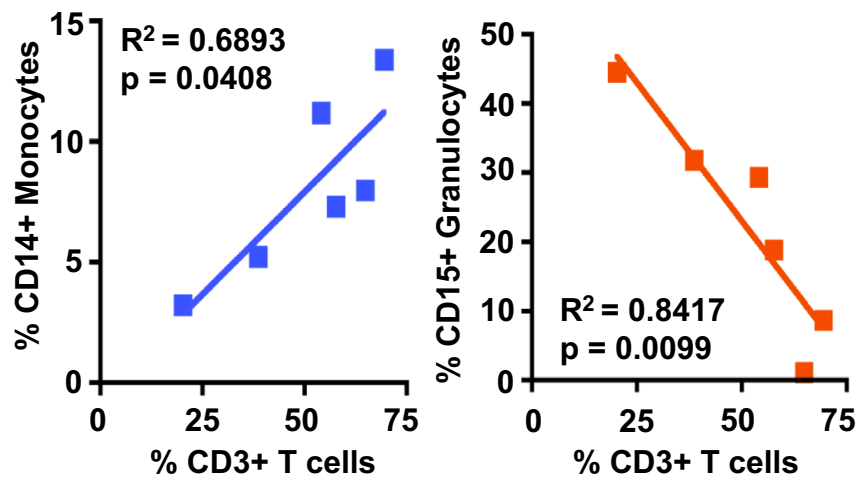

CD14+monocytic cells, and CD3 + TIL isolated from human ovarian cancer specimens and correlation of CD3 + TILs with either CD14+monocytic cells ( $\square$ ) or CD15+granulocytic cells $(\square)$ in human ovarian tumors $(n=6)$ 

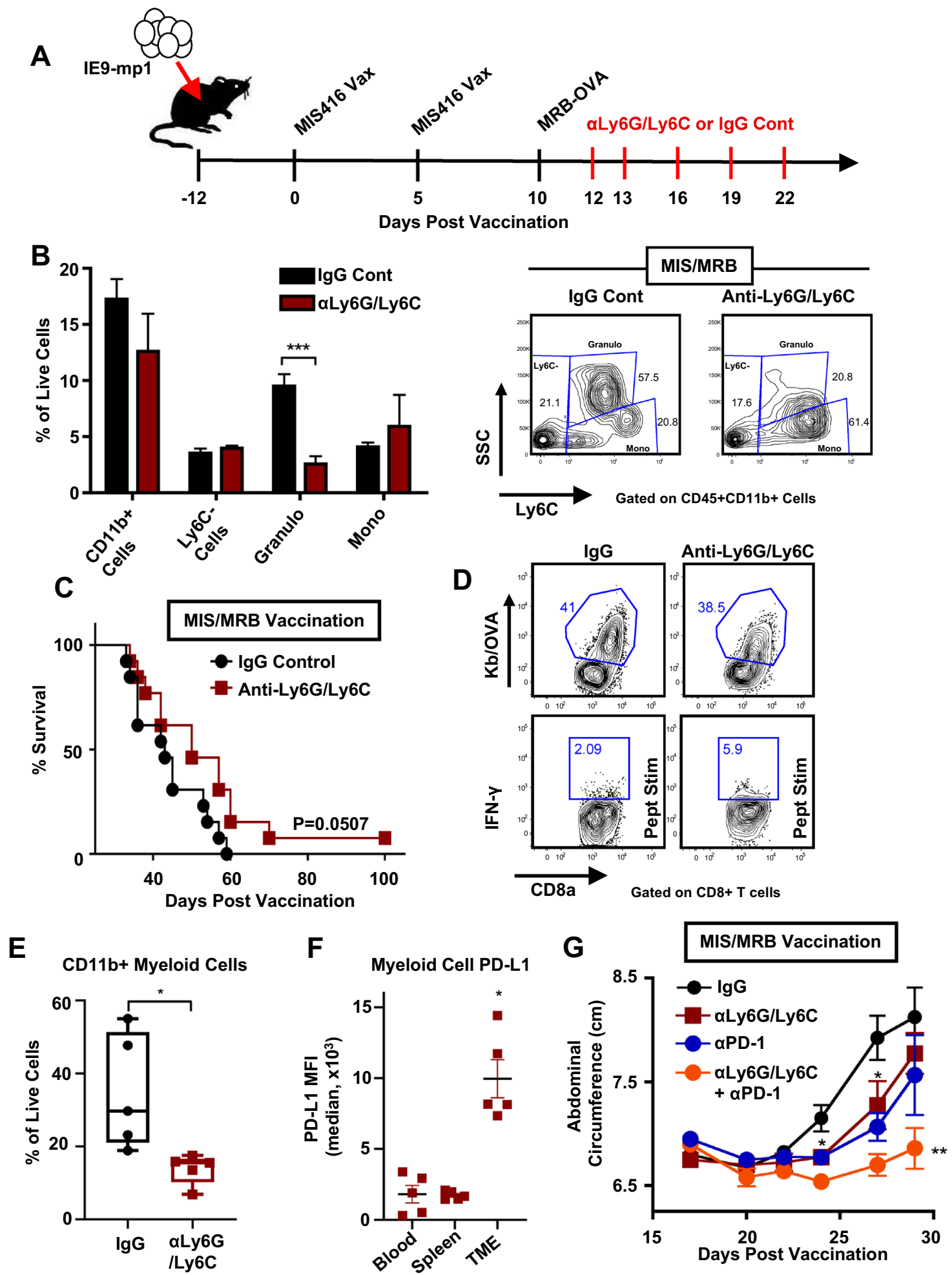

cell accumulation (Fig. 3b), suggesting that granulocytic cell infiltration in ovarian tumors might negatively impact the accumulation and/or persistence of CD3 + TIL.
Myeloid cell depletion improves prime/boost vaccination when combined with PD-1 checkpoint blockade

To examine whether myeloid cells were contributing to immune suppression and limiting the efficacy of MIS/MRB, 
४Fig. 4 Depletion of myeloid cells improves prime/boost vaccination and is further enhanced by PD-1 blockade. a Schematic representation of experimental design and treatment schedule for MIS/ MRB + anti-Ly6G/Ly6C or IgG Control. b Left Panel: Changes in indicated myeloid cell subsets in the peripheral blood following MIS/ $\mathrm{MRB}+\mathrm{IgG}$ or anti-Ly6G/Ly6C $(\mathrm{n}=5)$. Right Panel: Representative FACs plots demonstrating myeloid cell sub-setting used to assess changes in myeloid cells following MIS/MRB + IgG or anti-Ly6G/ Ly6C as shown in Left Panel. c Compiled survival data of IE9-mp1 tumor-bearing mice treated with MIS/MRB + IgG $(O)$ or anti-Ly6G/ Ly6C ( $(n=13)$. d Representative FACs plots showing matched $\mathrm{OVA}_{257-264}$ tetramer staining and ex vivo function of CD8+TALs following $\mathrm{OVA}_{257-264}$ peptide stimulation. Mice were treated with $\mathrm{MIS} / \mathrm{MRB}+\mathrm{IgG}$ or anti-Ly6G/Ly6C as indicated. e Frequency of CD11b+myeloid cells in the peritoneal TME following MIS/ $\mathrm{MRB}+\mathrm{IgG}$ or anti-Ly6G/Ly6C $(n=5)$. f PD-L1 MFI for residual $\mathrm{CD} 11 \mathrm{~b}+$ myeloid cells in the blood, spleen, and peritoneal TME following MIS/MRB + anti-Ly6G/Ly6C $(n=5)$. g IE9-mp1 tumor progression in mice following MIS/MRB combined with IgG (O), antiLy6G/Ly6C ( ), anti-PD-1 (O), or anti-Ly6G/Ly6C + anti-PD-1 (O ) $(n=3-5)$. Data presented as mean \pm SEM. Data in $(\mathbf{g})$ are from a single experiment

cellular depletion using an anti-Ly6G/Ly6C antibody was combined with MIS/MRB (Fig. 4a). MIS/MRB + anti-Ly6G/ Ly6C was well tolerated, however, 2/25 mice developed toxicity (weight loss, poor body condition) requiring euthanasia. As the RB6-8C5 antibody competes for binding with the IA8 anti-Ly6G antibody commonly used for cell phenotyping [11], effective myeloid cell depletion was assessed on circulating $\mathrm{CD} 11 \mathrm{~b}+$ myeloid cell subsets based on Ly6C expression and SSC profile (Fig. 4b). Anti-Ly6G/Ly6C treatment did not significantly impact the frequency of circulating CD11b + myeloid cells or monocytes but resulted in a significant reduction of Ly6C ${ }^{\text {lo }} \mathrm{SSC}^{\text {hi }}$ granulocytic cells (Fig. 4b). Anti-Ly6G/Ly6C delivery did not significantly improve survival of tumor-bearing mice (Fig. 4c) or reverse the dysfunction of tumor-specific CD8 + TALs following MIS/MRB (Fig. 4d) despite a more than 2.5-fold reduction in CD11b + myeloid cells in the TME (Fig. 4e). Residual CD11b + myeloid cells in the TME following anti-Ly6G/Ly6C depletion had elevated surface PD-L1 compared to myeloid cells in the blood and spleen (Fig. 4f) and while delivery of single-agent anti-Ly6G/Ly6C or antiPD-1 improved prime/boost vaccination, combining both antibody therapies with MIS/MRB resulted in a further increase in tumor control compared to either monotherapy (Fig. 4g). Together, these data point to a role for depletion of immunosuppressive myeloid cells as a strategy for enhancing the efficacy of immunotherapeutic combinations for ovarian cancer.

\section{Discussion}

While effective in pre-clinical models, cancer vaccines demonstrate only modest clinical efficacy in ovarian cancer [12]. However, technical advancements including improved antigen discovery [13] and approaches to bolster vaccine effectiveness [14] continue to drive interest in cancer vaccine development. In the current study, we identified strategies that enhance an already effective prime/boost cancer vaccine and which are complemented by ICB. The approach of using a robust cancer immunotherapy as a therapeutic screening platform has solid potential for identifying rational targets and mechanistic defects likely to augment anti-tumor immunity or synergize with existing therapies.

We identified that insufficient $T$ cell co-stimulation via the CD27/CD70 axis limited MIS/MRB efficacy. While CD27 co-stimulation delayed tumor growth, efficacy was further improved when vaccination was combined with antiPD-1 and anti-CD27. This is in line with clinical observations combining a CD27 agonist with PD-1 blockade, which led to a partial response or stable disease in 24 of 49 ovarian cancer patients (49\%), with intratumoral increases in PD-L1 expression and $\mathrm{CD} 8+\mathrm{T}$ cells correlating with improved outcome [15].

Similarly, reducing CD11b + myeloid cell accumulation in the TME using a depleting antibody, particularly following PD-1 blockade, also improved vaccine efficacy. In line with previous observations [11], Ly6G/Ly6C-specific antibody delivery led to granulocytic cell depletion, however, we cannot definitively rule out effects on additional Ly6G + or Ly6C + cells. A recent report from our group demonstrated that mature neutrophils can actively suppress $\mathrm{T}$ cells following exposure to ovarian cancer ascites [16] and this is further supported by the current study, where we observed tumor- infiltrating granulocytic cells to negatively correlate with CD3 + TILs in human ovarian cancer. Data from our pre-clinical model further suggest that suppressive myeloid cells can limit the activity of vaccine-elicited T cells. Given the suppressive function of intratumoral myeloid cells, strategies to limit tumor infiltration or re-polarize myeloid cells are being explored clinically [17]. Whether targeting discrete myeloid cell subsets leads to meaningful clinical benefit or will necessitate combination therapy remains to be determined. In this regard, the high PD-L1 expression on intratumoral myeloid cells observed in this study is consistent with data highlighting a mechanistic role for PD-L1 + myeloid cells in suppressing anti-tumor immunity and the response to ICB [18]. Importantly, the elevated PD-L1 on myeloid cells in the context of strong inflammatory signals in the TME elicited through prime/boost vaccination [8] suggests that therapies aimed at generating pro-inflammatory myeloid cells should consider the PD-L1 status of resulting myeloid cell subsets and whether such interventions will necessitate ICB to improve anti-tumor immunity.

Our findings highlight that while antibody-based therapies acting directly on $\mathrm{T}$ cells or indirectly via myeloid cell targeting can positively impact the anti-tumor $\mathrm{T}$ cell response, addition of PD-1 blockade further enhanced T cell activity in the TME. This point is strengthened by our 
previous observation that the dysfunction of tumor-specific CD8 + T cells in the TME following MIS/MRB was largely reversed when vaccination was combined with PD-1 blockade [8]. As the landscape of clinical cancer immunotherapies continues to develop, an improved understanding of how specific treatment combinations enhance and/or broaden the functionality of tumor-specific T cells will allow for refined therapeutic approaches based on an individual's tumor immune landscape.

Numerous ICB agents are being explored pre-clinically, are under clinical evaluation, or have received regulatory approval, and ICB is rapidly becoming part of the clinical standard of care. Therefore, identifying the appropriate context for combining emerging therapies with ICB is necessary to effectively design future clinical immunotherapy trials [19]. This will undoubtedly require improved pre-clinical tumor models that take into consideration tumor heterogeneity, variably immunogenic tumor landscapes, as well ICB resistance/failure. In this regard, we have previously reported that antigen loss variants (ALV) emerge in a subset of relapsing tumors isolated from mice treated with MIS/ MRB + anti-PD-1 in the setting of advanced ovarian cancer [8]. Outgrowth of ALV occurs during the immune evolution of tumors, and pre-clinical models of ALV represent valuable tools to understand how antigen/epitope spreading might sustain immune attack following antigen loss. Importantly, ALV emerged in all relapsed IE9-mp1 tumors following MIS/MRB + anti-PD-1/CD27 (unpublished data), indicating that (i) ALV prevent complete tumor eradication in this model, (ii) ALV outgrowth limits determination of the full therapeutic potential of MIS/MRB + anti-PD-1/CD27, thus preventing a clear understanding of to what degree CD27 agonism can improve the MIS/MRB + anti-PD-1 combination and (iii) any broadening of T cell responses to additional tumor antigens following therapy is not sufficient to sustain tumor attack in the context of ALV. Whether this is due to low tumor immunogenicity at relapse, limited tumor-specific $\mathrm{T}$ cell repertoire, or upregulation of additional resistance mechanisms remains an active area of investigation. In this regard, identification of tumor reactive $\mathrm{T}$ cells with specificity for a broader array of tumor antigens (present either at baseline or which may become detectable through epitope spreading following prime/boost vaccination) could allow for additional vaccinations to counteract tumor immune evasion following single antigen loss, provided resistance mechanisms in the TME do not significantly hinder durable T cell activity.

While effective in some cancers, clinical responses to immunotherapy in ovarian cancer have been less frequent. However, complete clinical responses in some patients combined with clear prognostic value of intraepithelial CD8 + TIL [20] suggest that ovarian tumors are immunologically positioned to respond. Using a prime/boost cancer vaccine to elicit robust expansion of tumor-specific $\mathrm{CD} 8+\mathrm{T}$ cells, we have identified therapies that rationally combine with cancer vaccines and/or checkpoint inhibitors. These findings underscore that generating durable tumor attack by $\mathrm{T}$ cells requires a multi-faceted approach, which may need to co-evolve with tumor progression and/or a shifting intratumoral immune landscape during treatment.

\section{Methods}

\section{Tumor challenge and Immunization}

Mice were challenged with $10^{7}$ IE9-mp1 in $500 \mu$ PBS by IP injection. Mice were immunized beginning 12 days post-tumor implantation. Preparation and administration of the MIS416+OVA and MRB-OVA vaccines used in this study have been previously detailed [8]. As IE9-mp1 tumors develop as widely disseminated disease within the peritioneal cavity, tumor progression was tracked based on increase in abdominal circumference due to accumulation of peritoneal ascites. This approach is based on our previous findings using serial magnetic resonance imaging (MRI) to track tumor growth [8], where accumulation of ascites was observed to closely parallel increases in solid tumor burden and disease progression. Mice were euthanized when abdominal circumference was $\geq 10 \mathrm{~cm}$ or when mice exhibited reduced body condition due to tumor progression.

\section{In vivo Monoclonal antibody delivery}

Monoclonal antibodies or relevant IgG controls were delivered to mice by IP injection $(200 \mu \mathrm{g} / \mathrm{mouse} / \mathrm{dose}$ in $200 \mu \mathrm{l}$ of PBS). Gr-1 depletion (Clone RB6-8C5) was commenced two days after Maraba boosting and was delivered on two consecutive days and then every third day for a total of 5 doses. For PD-1 blockade, anti-PD-1 (Clone RMP1-14) was delivered every third day to mice beginning the day of Maraba boosting for a total of 5 doses. Anti-CD27 agonist antibody (Clone AT124-1) was administered on days 3 and 7 following MRB-OVA boosting.

A detailed description of additional methods has been included as a supplemental file.

Supplementary Information The online version contains supplementary material available at https://doi.org/10.1007/s00262-021-02936-1.

Acknowledgements This work was supported by the Ovarian Cancer Research Alliance Grant 326870, the Roswell Park Alliance Foundation, the NCI funded RPCI-UPCI Ovarian Cancer SPORE P50CA159981-01A1, U01 CA233085-01A1, R01CA188900, the P30CA016056 Grant involving the use of Roswell Park Cancer Institute's shared resources including the Pathology Resource Network, Genomics Shared Resource, Flow Cytometry Core, and Laboratory 
Animal Resources. We would like thank Ariel Francois for breeding mice used in this study.

Author contributions AJRM designed the study, conducted experiments, collected, analyzed, and interpreted data, drafted the manuscript and revised the final version. CE bred animals used in the study, contributed to data review and interpretation, and critically reviewed the final version of the manuscript. AM conducted experiments. KLS conducted experiments, collected data, and critically reviewed the final version of the manuscript. KS purified viruses used in the study, contributed to experimental design, and critically reviewed the final version of the manuscript. AL contributed to the design of the study, data review and interpretation, and drafting/revision of the manuscript. BHS reviewed data, contributed to data interpretation and critically reviewed the final version of the manuscript. TK provided insight towards the use of the CD27 agonist antibody, contributed to data review and interpretation, and critically reviewed the manuscript. GW advised on the use of the MIS416 adjuvant, critically reviewed data, provided input related to study design, and reviewed the manuscript. BL provided input related to the use of Maraba as a boosting agent, reviewed the data and assisted with interpretation, and critically reviewed the manuscript. DK contributed to experimental design, review and interpretation of data, and critical review of the manuscript. KO designed the study, reviewed and interpreted the data, and drafted and revised the final manuscript.

Funding This work was supported by the Ovarian Cancer Research Alliance Grant 326870:(AJRM), the Roswell Park Alliance Foundation, the NCI funded RPCI-UPCI Ovarian Cancer SPORE P50CA15998101A1, U01 CA233085-01A1 (KO and DK), R01CA188900, R03CA223623 (DK), the P30CA016056 Grant involving the use of Roswell Park Cancer Institute's shared resources including the Pathology Resource Network, Genomics Shared Resource, Flow Cytometry Core, and Laboratory Animal Resources

Availability of data and materials Data used or analyzed during the current study available from the corresponding author on reasonable request

\section{Declarations}

Conflict of interest K Stephenson is an employee of Turnstone Biologics, who hold IP for Maraba Virus. T Keler is the Founder, Executive Vice President, and Chief Scientific Officer of Celldex Therapeutics, who provided the anti-CD27 antibody for these studies. G Webster is the Chief Scientific Officer of Innate Immunotherapeutics, who hold IP for MIS416. B Lichty is an inventor and shareholder of Turnstone Biologics, who hold IP for Maraba Virus. K Odunsi received research funding from Astra Zeneca and Tessaro; and is a co-founder of Tactiva Therapeutics. All other authors have no financial conflicts of interest to disclose

Ethical approval For animal studies, all performed experiments and procedures were reviewed and approved by the Roswell Park IACUC prior to conducting experiments. For experiments involving human tumor specimens, samples were received in the laboratory from Roswell Park tissue procurement under approved protocol I215512 which allows experimental use of patient tissue specimens for research.

\section{References}

1. Kalbasi A, Ribas A (2020) Tumour-intrinsic resistance to immune checkpoint blockade. Nat Rev Immunol 20:25-39. https://doi.org/10.1038/s41577-019-0218-4

2. McGray AJR, Bramson J (2017) Adaptive resistance to cancer immunotherapy. Adv Exp Med Biol 1036:213-227. https://doi. org/10.1007/978-3-319-67577-0_14

3. Jenkins RW, Barbie DA, Flaherty KT (2018) Mechanisms of resistance to immune checkpoint inhibitors. Br J Cancer 118:916. https://doi.org/10.1038/bjc.2017.434

4. González-Martín A, Sánchez-Lorenzo L (2019) Immunotherapy with checkpoint inhibitors in patients with ovarian cancer: still promising? Cancer 125(Suppl 24):4616-4622. https://doi.org/ $10.1002 /$ cncr. 32520

5. Odunsi KK, Matsuzaki JJ, Karbach JJ et al (2012) Efficacy of vaccination with recombinant vaccinia and fowlpox vectors expressing NY-ESO-1 antigen in ovarian cancer and melanoma patients. Proc Natl Acad Sci U S A 109:5797-5802. https://doi. org/10.1073/pnas.1117208109

6. Odunsi K, Matsuzaki J, James SR et al (2014) Epigenetic potentiation of NY-ESO-1 vaccine therapy in human ovarian cancer. Cancer Immunol Res 2:37-49. https://doi.org/10.1158/23266066.CIR-13-0126

7. Szender JB, Papanicolau-Sengos A, Eng KH et al (2017) NYESO-1 expression predicts an aggressive phenotype of ovarian cancer. Gynecol Oncol 145:420-425. https://doi.org/10.1016/j. ygyno.2017.03.509

8. McGray AJR, Huang R-Y, Battaglia S et al (2019) Oncolytic Maraba virus armed with tumor antigen boosts vaccine priming and reveals diverse therapeutic response patterns when combined with checkpoint blockade in ovarian cancer. J Immunother Cancer 7:189. https://doi.org/10.1186/s40425-019-0641-x

9. Hintzen RQ, Lens SM, Lammers K et al (1995) Engagement of CD27 with its ligand CD70 provides a second signal for $\mathrm{T}$ cell activation. J Immunol 154:2612-2623

10. Starzer AM, Berghoff AS (2020) New emerging targets in cancer immunotherapy: CD27 (TNFRSF7). ESMO Open. https:// doi.org/10.1136/esmoopen-2019-000629

11. Ribechini E, Leenen PJM, Lutz MB (2009) Gr-1 antibody induces STAT signaling, macrophage marker expression and abrogation of myeloid-derived suppressor cell activity in BM cells. Eur J Immunol 39:3538-3551. https://doi.org/10.1002/eji. 200939530

12. Martin Lluesma S, Wolfer A, Harari A, Kandalaft L (2016) Cancer vaccines in ovarian cancer: how can we improve? Biomedicines 4:10. https://doi.org/10.1038/nrc3973

13. Liu S, Matsuzaki J, Wei L et al (2019) Efficient identification of neoantigen-specific T-cell responses in advanced human ovarian cancer. J Immunother Cancer 7:156. https://doi.org/10.1186/ s40425-019-0629-6

14. Ott PA, Hu Z, Keskin DB et al (2017) An immunogenic personal neoantigen vaccine for patients with melanoma. Nature 547:217-221. https://doi.org/10.1093/nar/gkn202

15. Sanborn RE, Pishvaian MJ, Callahan MK et al (2018) AntiCD27 agonist antibody varlilumab (varli) with nivolumab (nivo) for colorectal (CRC) and ovarian (OVA) cancer: phase (Ph) 1/2 clinical trial results. J Clin Oncol 36:3001-3001. https://doi.org/ 10.1200/JCO.2018.36.15_suppl.3001

16. Singel KL, Emmons TR, Khan ANH et al (2019) Mature neutrophils suppress $\mathrm{T}$ cell immunity in ovarian cancer microenvironment. JCI Insight. https://doi.org/10.1172/jci.insight.122311

17. Jahchan NS, Mujal AM, Pollack JL et al (2019) Tuning the tumor myeloid microenvironment to fight cancer. Front Immunol 10:1611. https://doi.org/10.3389/fimmu.2019.01611 
18. Tang H, Liang Y, Anders RA et al (2018) PD-L1 on host cells is essential for PD-L1 blockade-mediated tumor regression. J Clin Invest 128:580-588. https://doi.org/10.1172/JCI96061

19. Nakhoda SK, Olszanski AJ (2020) Addressing recent failures in immuno-oncology trials to guide novel immunotherapeutic treatment strategies. Pharmaceut Med 34:83-91. https://doi.org/10. 1007/s40290-020-00326-z

20. Sato E, Olson SH, Ahn J et al (2005) Intraepithelial CD8+ tumorinfiltrating lymphocytes and a high $\mathrm{CD} 8+$ /regulatory $\mathrm{T}$ cell ratio are associated with favorable prognosis in ovarian cancer. Proc Natl Acad Sci U S A 102:18538-18543. https://doi.org/10.1073/ pnas.0509182102

Publisher's Note Springer Nature remains neutral with regard to jurisdictional claims in published maps and institutional affiliations. 